\title{
Fungal sinusitis with ocular involvement: Case report
}

\author{
Achyut N. Pandey ${ }^{1^{*}}$, Anil Kakde $^{2}$ \\ ${ }^{1}$ Department of Ophthalmology, VCSG Medical College and Research Institute, Srinagar-Garhwal, Uttarakhand, India; \\ *Corresponding Author: achyutpandey@gmail.com \\ ${ }^{2}$ Eye Q Super Speciality Eye Hospital, Gurgaon, India
}

Received 7 October 2013; revised 5 November 2013; accepted 3 December 2013

Copyright (C 2013 Achyut N. Pandey, Anil Kakde. This is an open access article distributed under the Creative Commons Attribution License, which permits unrestricted use, distribution, and reproduction in any medium, provided the original work is properly cited. In accordance of the Creative Commons Attribution License all Copyrights (c) 2013 are reserved for SCIRP and the owner of the intellectual property Achyut N. Pandey, Anil Kakde. All Copyright (C) 2013 are guarded by law and by SCIRP as a guardian.

\section{ABSTRACT}

Rhino-orbital-cerebral mucormycosis (ROCM) is an acute, often fatal, fungal infection caused by members of the class Zygomycetes and the order Mucorales. The genus Rhizopus accounts for most cases of ROCM. The disease is characterized by fungal hyphal invasion of blood vessels resulting in thrombosis and infarction of the nasal, paranasal sinus, orbital, and cerebral tissues. The most commonly associated condition is diabetes mellitus; other associated conditions include immunocompromised states, renal disease, deferoxamine use and acidotic states. The most frequent sites of infection are pulmonary, rhinocerebral, cutaneous and disseminated. Rhino-orbital and Rhino-cerebral are two forms of the disease. As such the condition is a medical emergency. Early recognition and treatment are essential because it may lead to death in a few days. CROP usually begins in the palate or paranasal sinuses and rapidly spreads to the orbital contents. Proptosis, loss of vision and ophthalmoplegia occur and death from cerebral involvement commonly ensues. The fungus tends to invade arteries and cause thrombosis and tissue infarction. Rhizopus is the most commonly isolated genus in CROP, accounting for almost all cases. The diagnosis can be strongly suspected by the characteristic clinical manifestations. Therapy includes the treatment of the underlying disease, surgical excision of the necrotic tissue containing fungal elements and the systemic administration of amphotericin-B. Here we report the clinical features of a 32-years-old man presented mucormycosis.
Keywords: Diabetes Mellitus; Mucormycosis; Rhizopus spp.

\section{CASE REPORT}

A 32-years-old male patient presented a history of pain in the nose and defective vision in the left eye since one week. The pain was not relieved. He was apparently all right one week prior. He was a diabetic incidientally found one week back only. On admission, he was found to be a afebrile, conscious and well oriented to the time and place, with a BP of $140 / 90 \mathrm{mmHg}$, a pulse of $82 / \mathrm{min}$ and with a swelling on the nose. Vision is in RE 6/18 and LE 6/HM. On examination patient is found to have convergent squint. Extra ocular movements were restricted in all six gazes, indicating the involvement of 3, 5 and 6 cranial nerve (Figure 1). Corneal sensation is decreased in BE .There was no proptosis presented. IOP is in RE 22.4 and LE 18.9. People in both eyes were round, reacting to light both direct and consensual light reaction. Pt was dilated for fundus examination, and BE fundus found to be normal. His fasting blood sugar was 296 $\mathrm{mg} / \mathrm{dl}$, sodium was $12 \mathrm{mEq} / \mathrm{L}$, potassium was $2.5 \mathrm{mEq} / \mathrm{L}$., urea was $47 \mathrm{mg} / \mathrm{dl}$, creatinine was $0.9 \mathrm{mg} / \mathrm{dl}$ and haemoglobin was $11.7 \mathrm{gm} \%$. All other lab investigations are found to be normal. His nasal swab and maxillary and ethmoid sinus curettages were received in the microbiology laboratory for $\mathrm{KOH}$ mounts and fungal cultures to check for mucormycosis. The microscopic examination of the biopsy material and the nasal discharge was done in $10 \%$ $\mathrm{KOH}$ wet mounts. It showed the characteristic broad, aseptate, branched hyphae (Figures 2, 3). The fungus was grown on Sabouraud's dextrose agar at $37^{\circ} \mathrm{C}$. A microscopic examination of the growth which was done in a lactophenol cotton blue mount, revealed that it was Rhizopus (Figure 4). Further patient is advised for CT scan orbit and para nasal sinuses for orbital or cerebral spread 


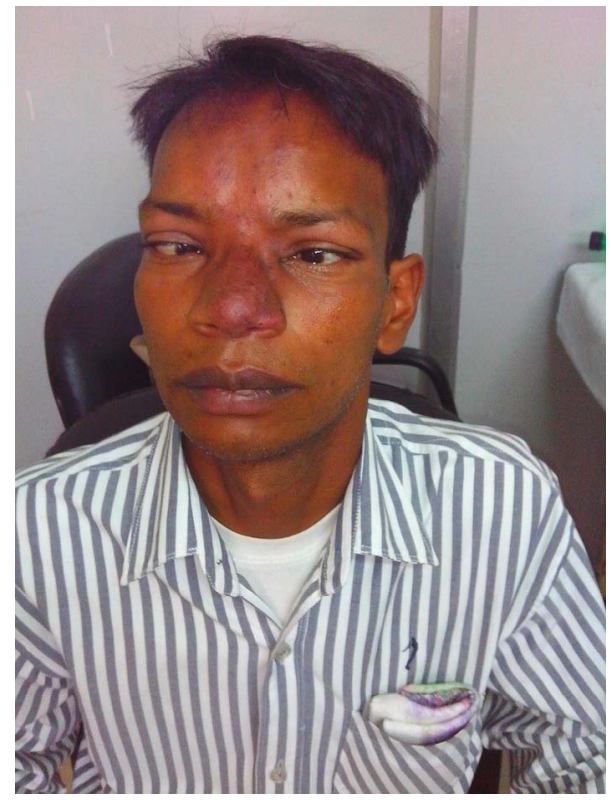

Figure 1. Patient with convergent squint, EOM restriction and swollen nose.

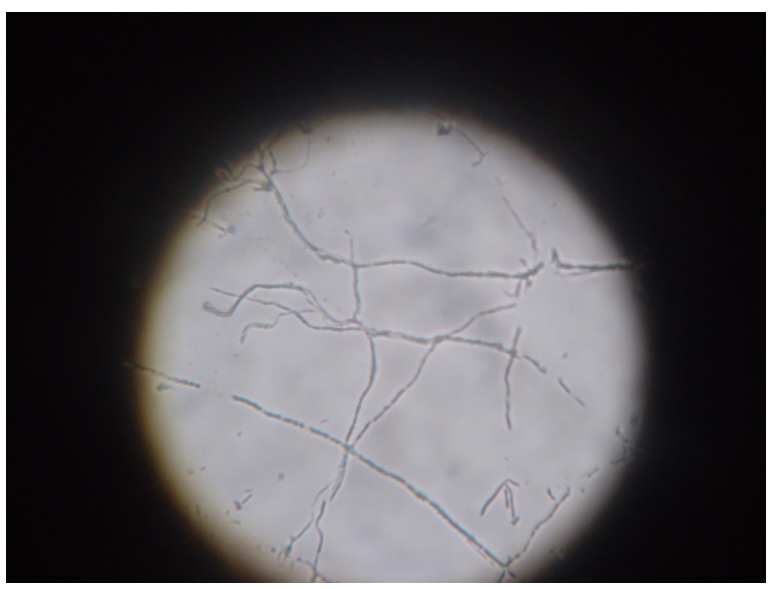

Figure 2. $\mathrm{KOH}$.
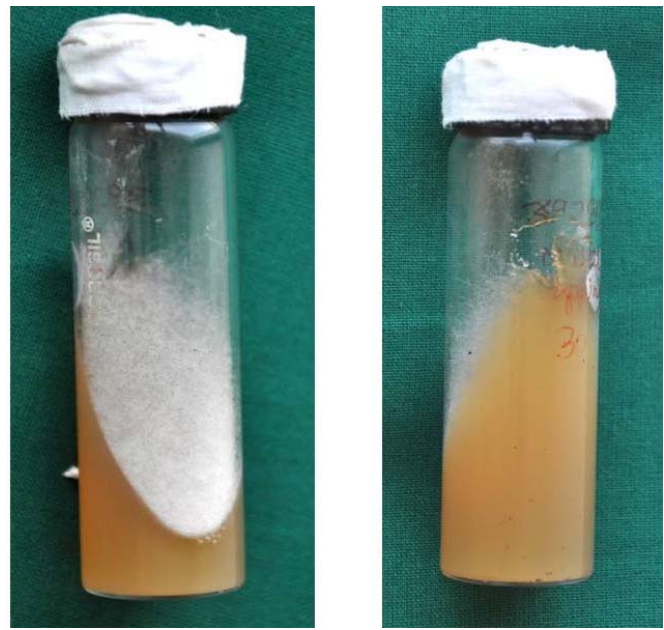

Figure 3. Growth on SDA.

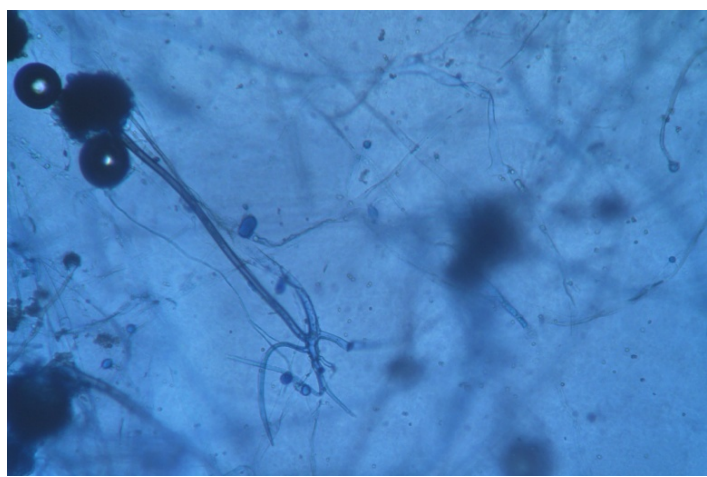

Figure 4. Lactophenol cotton blue.

(Figure 5). CT scan was found to be normal. The patient was started on an intravenous insulin infusion and amphotericin B at $0.3 \mathrm{mg} / \mathrm{kg} /$ day and this was gradually increased to $1 \mathrm{mg} / \mathrm{kg} /$ day with the monitoring of the serum electrolytes and the renal functions.

\section{DISCUSSION}

Mucormycosis are a group of invasive infections which are caused by filamentous fungi of the order, $\mathrm{Mu}$ corales of the Mucoraceae Family. Rhino-orbital mucormycosis is an aggressive, angioinvasive fungal infection which is seen inimmunocompromised hosts. The risk factors are poorly controlled Diabetes mellitus, haematological malignancies and a prolonged corticosteroid treatment. The infections which are caused by members of the order mucorales are primarily opportuneistic infections and they represent the third leading cause of invasive fungal infections following Aspergillus and Candida species. The most frequently isolated species is Rhizopus oryzae, followed by Rhizopus microsporeus and Absidia corymbifera [1].

Rhino-Orbital Mucormycosis (ROM) is a rare disease with an overall prevalence in $0.15 \%$ of the diabetics. However, rhino-orbital-cerebral mucormycosis, as a presenting manifestation, is rare [2]. Despite the advances in the diagnosis and treatment, a high mortality rate of $30 \%$ - 70\% still exists for this disease. Death may occur within two weeks in untreated or unsuccessfully treated patients. It is an acute opportunistic infection which is caused by a broad, nonseptate, saprophytic fungus which is found in soil, air, bread mould, rotten fruit and vegetables.It can be cultured from the mouth, nasal tract, throat and the faeces of healthy persons. The fungus belongs to the Phycomycetes class, whose most common genera are Mucor, Rhizopus, Absidia and Basidiobolus. Contact with the micro organism occurs through spore inhalation. The infection spreads along the vascular and neuronal structures and it infiltrates the walls of the blood vessels. It causes erosion of the bony walls of the ethmoid sinuses and it may spread into the orbit and the retro- 


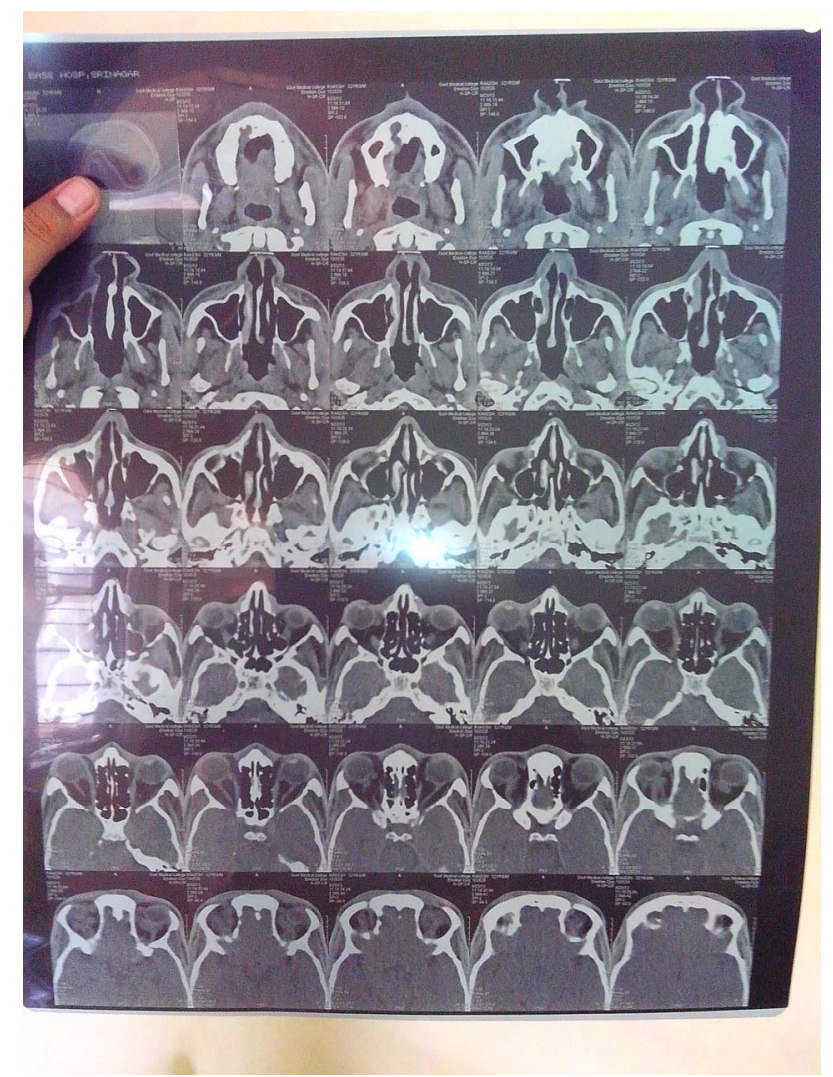

Figure 5. CT scan.

orbital area and in the brain (cerebro-rhino-orbital mucormycosis). Death may occur due to the cerebral abscesses. The infection with this organism usually complicates any underlying chronic disease, as in our patient. Commonly, mucormycosis attacks people with compromised immune systems. The reduced ability of serum to bind iron at a low $\mathrm{pH}$ may be the basic defect in the body's defense systems. The high iron, glucose-rich acid milieu facilitates fungal growth. The human resistance to fungal infections rests on the body's ability to restrict the availability of iron to the invading fungus, by binding it to proteins such as apotransferrin. The fungal hyphae produce a substance called rhizoferrin, which binds iron avidly. This iron-rhizoferrin complex is then taken up by the fungus and it becomes available for the vital intracellular processes. Diabetic patients are predisposed to mucormycosis because of the decreased ability of their neutrophils to phagocytose and adhere to the endothelial walls. Furthermore, the acidosis and hyperglycaemia provide an excellent environment for the fungus to grow [3]. In this case, the patient was a known diabetic and later on, he had developed ketosis. Initially, he had presented with pain in his nose which was there since seven days, which had progressed to Rhino-Orbital Mucormycosis (ROM) within the next five days. It was followed by a periorbital swelling and an inability to open the mouth (facial weakness). The prognosis of mucormycosis has markedly improved over the past 30 years, with a $90 \%$ survival rate [2]. The factors which are related to a poor survival are delays in the diagnosis and treatment. Mucormycosis, also known as Zygomycosis or Phycomycosis, was first described by Paultauf in 1885, who coined the term "mycosis mucorina". In 1943, three cases of rhinocerebral mucormycosis were reported in diabetic patients. The most pathogenic species of the family, Mucoraceae is Rhizopus. Rhizopus oryzae is the predominant pathogen which accounts for $60 \%$ of all the forms and $90 \%$ of the rhinocerebral cases [4]. In this case also, we isolated the Rhizopus oryzae species. Roden et al., [5] who reviewed 929 reports of zygomycosis in the English-language literature since 1885, found co-existing diabetes in $36 \%$ of the patients. Zygomycosis had caused death in $44 \%$ of them. However, Chakrabarti et al., [6] who analyzed 178 cases of Zygomycosis in hospitalized patients in northern India, found co-existing, uncontrolled diabetes in $73.6 \%$ of the patients. Moreover, Schwartz et al., [7] noticed that Cerebro-Rhino-Orbital Phycomycosis (CROP) which was mainly caused by Rhizopus spp., occurred predominantly in the individuals with diabetic ketoacidosis. CROP leads to proptosis, loss of vision, ophthalmoplegia and death which results from a cerebral involvement.

\section{CONCLUSION}

Mucormycosis remains a severe infectious disease in diabetic patients and it is characterized by a high mortality rate. The clinical diagnosis is often difficult and it gets delayed. It should be underlined that in diabetics, physicians should always pay special attention to the infections within the facial skeleton, especially which do not respond to antibiotic therapy. Aggressive diagnostic procedures are required for histo-microbiological studies to confirm this disease. An early diagnosis, combined with medical and surgical treatments, is necessary to improve the outcome.

\section{ACKNOWLEDGEMENTS}

The author is thankful to his patient and family. The author also acknowledges the valuable support of divyam pandey.

\section{REFERENCES}

[1] Mallis, A, Mastronikolis, S.N., Naxakis, S.S. and Papadas, A.T. (2010) Rhinocerebral mucormycosis: An update. European Review for Medical and Pharmacological Sciences, 14, 987-992.

[2] Mohd, H., Syed, M., Sameena, S. and Riyaz, S. (2011) Rhino-orbital-mucormycosis as presenting manifestation of gestational diabetes mellitus. Indian Journal of Endo- 
crinology and Metabolism, 15, 65-66.

[3] Mathebula, S.D. (2006) Case report: Rhino-orbital mucormycosis. The South African optometrist, 65, 78-81.

[4] Lamia, A., Badreddine, K., Hanene, T., Kanoun, F., Goubontini, A., Mnif, E., Zouiten, F., Chaker, E. and Chaabane, T. B. (2008) Mucormycose: A propos de quatre observations. La Tunisie Medicale, 86, 165-168.

[5] Roden, M.M., Zaoutis, T.E., Buchanan, W.E., et al. (2005) Epidemiology and outcome of zygomycosis: A review of 929 reported cases. Clinical Infectious Diseases, 41, 634-
653. http://dx.doi.org/10.1086/432579

[6] Chakrabarti, A., Das, A., Mandal, J., et al. (2006) The rising trend of invasive Zygomycosis in patient with uncontrolled diabetes mellitus. Medical Mycology, 44, 335342. http://dx.doi.org/10.1080/13693780500464930

[7] Schwart, J.N., Donnelly, E.H. and Kintworth, G.K. (1977) Ocular and orbital phycomycosis. Survey of Ophthalmology, 22, 3-28.

http://dx.doi.org/10.1016/0039-6257(77)90025-X 